\title{
Alpha7 Nicotinic Acetylcholine Receptor Down Regulation Impairs Mitochondrial Function in Streptozotocin-induced Sporadic Alzheimer's Disease Model in Rats
}

\author{
Niraj Kumar Singh, Debapriya Garabadu* \\ Division of Pharmacology, Institute of Pharmaceutical Research, GLA University, Mathura, Uttar Pradesh, INDIA.
}

\begin{abstract}
Background/Aim: Alzheimer's disease (AD), a type of neurodegenerative disorder, possesses significant memory loss as one of the cardinal manifestations. The pathophysiology of $A D$ includes increased accumulation of $A \beta$, degeneration of cholinergic activity and mitochondrial dysfunction. Nicotinic acetylcholine receptors (nAChRs) especially alpha7 nicotinic acetylcholine receptors ( $\alpha 7 \mathrm{nAChRs)}$ are widely distributed in brain and associated with memory function. Further, we explored the correlation between the mitochondrial dysfunction and the expression level of $\alpha 7 \mathrm{nAChRs}$ in STZ challenged brain regions of rats. Materials and Methods: The STZ group rats received intracerebroventricular infusion of STZ $(3 \mathrm{mg} / \mathrm{kg})$ on D-1 and D-3 of experimental design of 18 days. Behavioral parameters ware investigated using MWM and Y-maze test paradigm. Further, biochemical analysis was assessed in all three regions of rats. Results: STZ administration caused significant impairment in memory and learning of rats MWM and $\mathrm{Y}$-maze test paradigm. There was significant decrease in level expression of $\alpha 7$ $\mathrm{nAChRs}$ and cholinergic functions in terms of elevated AChE activity and decreased ACh level and ChAT activity in rat hippocampus, pre-frontal cortex and amygdala. Further, STZ administration significantly attenuated the mitochondrial function, integrity and bioenergetics in all the selected brain regions. Interestingly, the intracerebroventricular infusion of STZ increased A $\beta$ level in all the rat brain regions. Conclusion: The $\alpha 7 \mathrm{nAChR}$ down regulation may form a basis to cognitive deficits along with cholinergic dysfunction, $\mathrm{A} \beta$ accumulation and mitochondrial dysfunction in memory sensitive rat brain regions. Thus, $\alpha 7 \mathrm{nAChR}$ could be an alternative and potential target in the management of AD.
\end{abstract}

Key words: Alzheimer's disease, Streptozotocin, $\alpha 7$ nAChR, Mitochondria, Acetylcholine, Hippocampus.

\section{INTRODUCTION}

Alzheimer's disease (AD), a type of neurodegenerative disorder, possesses significant memory loss as one of the cardinal manifestations. The pathophysiology of AD includes increased accumulation of amyloid beta (A $\beta$ ), degeneration of cholinergic activity, dysregulation in the calcium homeostasis and mitochondrial dysfunction. ${ }^{1,2}$ It is expected that approximately 115 million people will be affected with $\mathrm{AD}$ throughout the globe by $2050.3,4$ Several drugs have been considered in the management of AD however, their use is restricted due to evidence of severe adverse effects. ${ }^{5}$ Hence, there is a need to develop novel therapeutic target to manage the progress of AD.

The nicotinic acetylcholine receptors (nAChRs) especially alpha7 ( $\alpha 7)$ are expressed throughout the brain and they are also associated in the process of memory formations. Utsugisawa et al. reported down regulation of the $\alpha 7 \mathrm{nAChR}$ in the brain of the subjects in postmortem study. ${ }^{6}$ It has
Submission Date: 07-06-2020; Revision Date: 13-10-2020; Accepted Date: 16-12-2020

DOI: 10.5530/ijper.55.1.17 Correspondence: Dr. Debapriya Garabadu Associate Professor, Division of Pharmacology, Institute of Pharmaceutical Research, GLA University, Mathura-281406, Uttar Pradesh, INDIA.

Phone no: +91 8853139229

Email id: debapriya. garabadu@gla.ac.in

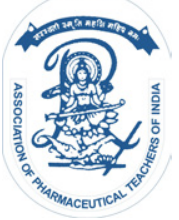

www.ijper.org 
been well reported that progressive loss of cognitive ability is associated with early deficits in the $\alpha 7 \mathrm{nAChRs.}{ }^{7}$ Literature review suggests that the $\alpha 7 \mathrm{nAChRs}$ plays a critical role in the physiology of $A \beta$ accumulation and also contribute in the pathophysiology of $\mathrm{AD}^{8,9} \mathrm{~A} \beta$ modulates postsynaptic nAChRs function especially $\alpha 7 \mathrm{nAChR}^{10}{ }^{10}$ It has been reported that neuronal inflammation may contribute to the reduction in $\alpha 7$ $\mathrm{nAChR}$ density in rodent brains and is associated with $A \beta$ deposition and memory impairments. ${ }^{11,12}$ On contrary, the activation of $\alpha 7 \mathrm{nAChR}$ attenuates $A \beta$ toxicity and exerts neuroprotection in the experimental studies. ${ }^{13,14}$ The activation of $\alpha 7 \mathrm{nAChR}$ also facilitates the activity of acetylcholine (ACh) in brain. ${ }^{15}$ Further, selective $\alpha 7$ nAChR agonist improves memory formation/function in the rodents. ${ }^{16,17} \mathrm{It}$ has been reported that the $\alpha 7 \mathrm{nAChRs}$ in the plasma membrane influence $\mathrm{Ca}^{2+}$ accumulation, voltagedependent anion channel (VDAC)-mediated $\mathrm{Ca}^{2+}$ transport and mitochondrial permeability and regulate the mitochondrial-dependent cell apoptosis. ${ }^{18}$ It is also suggested that the $\alpha 7 \mathrm{nAChR}$ could possess a major clinical and pharmacological implications in the management of $\mathrm{AD} .^{19}$

It has been observed that the intracerebroventricular (ICV) injection of streptozotocin (STZ) alters behavioral functions including memory impairements ${ }^{20}$ and neurochemical functions ${ }^{21,22}$ similar to AD condition. It is demonstrated that ICV-STZ leads to mitochondrial dysfunction ${ }^{23}$ with deficiency in cholinergic activity ${ }^{24}$ in AD-like rodents. Tota $e t$ al. reports significant reduction in the expression level of $\alpha 7 \mathrm{nAChRs}$ with concomitant dysfunction in mitochondria and cholinergic activity in the STZ-challenged rodent brains. ${ }^{25}$ However, there is no report on the brain $\alpha 7 \mathrm{nAChRs}$ level in STZchallenged animals.

Therefore, present study was undertaken to explore the level of expression of $\alpha 7 \mathrm{nAChRs}$ and, function, integrity and bioenergetics of mitochondria in the memorysensitive brain areas of STZ-challenged rats.

\section{MATERIALS AND METHODS}

\section{Animals}

Swiss albino wistar male rats $(200 \pm 20 \mathrm{~g})$ were collected from the animal house of GLA University, Mathura and Uttar Pradesh. The rats were divided into three equal-sized groups $(n=6)$ and housed at ambient condition (temperature $25 \pm 1^{\circ} \mathrm{C}$, relative humidity $50 \pm 5 \%$ and light/dark cycle $12: 12 \mathrm{~h}$ ) in poly-acrylic cages. Animals received their standard pellet feed (Lipton India, Ltd., Mumbai) and water ad libitum in the experimental study. All the animals were fasted for $14 \pm$ $1 \mathrm{~h}$ before experimentation, but water was supplied in sufficient quantity. The study deign was approved from the institute for experiment on animals (GLAIPR/ CPCSEA/IAEC/P'Col/2016/01).

\section{Reagents and Chemicals}

All the used chemicals in the experimentation were of analytical grade and available commercially. STZ, TMRM and Amplex red assay kit for acetylcholinesterase (AChE) and ACh were procured from Sigma (St. Louis, MO, USA) while beta-actin was purchased from Abcam Plc., Cambridge, USA.

\section{Experimental Design}

The experimental design consisted of eighteen days study (Figure 1). Rats were randomly allocated into 3 groups (Control, Sham and STZ) having 6 animals in each group. ICV-STZ was injected on Day (D)-1 and D-3 of experimental protocol only to STZ group rats. Sham group animals received artificial cerebrospinal fluid (aCSF). The Morris Water Maze (MWM) test paradigm was carried out for 5 successive days (from D-14 to 18) of the experimental design. Further, Y-maze test paradigm was carried out on D-18 with a one hour of lag between two test paradigms. All the behavior parameters were record and computed with ANY-Maze ${ }^{\mathrm{TM}}$ video-tacking software (Version-4.69, USA). After that decapitation method was used to sacrifice all the rats. The brain were removed and micro-dissected into hippocampus (HIP), prefrontal cortex (PFC) and amygdale (AMY) and used for further biochemical estimation..$^{26,27}$

\section{ICV Injection of STZ}

Overnight fasted rat was anesthetized with intraperitoneal injection of sodium pentobarbital (40 $\mathrm{mg} / \mathrm{kg}$ ). After that an incision was made on the scalp and the head was positioned in a stereotaxic apparatus (Stoelting, USA). On each sides of lateral ventricle, two holes $(1.5 \mathrm{~mm}$ depth) were drilled $(0.8 \mathrm{~mm}$ posterior, $\pm 1.5 \mathrm{~mm}$ medio-lateral relative to bregma and $3.8 \mathrm{~mm}$ drso-ventral below to dura) ${ }^{26}$ Freshly prepared STZ solution in aCSF $(47 \mathrm{mM} \mathrm{NaCl} ; 2.9 \mathrm{mM} \mathrm{KCl} ; 1.6 \mathrm{mM}$

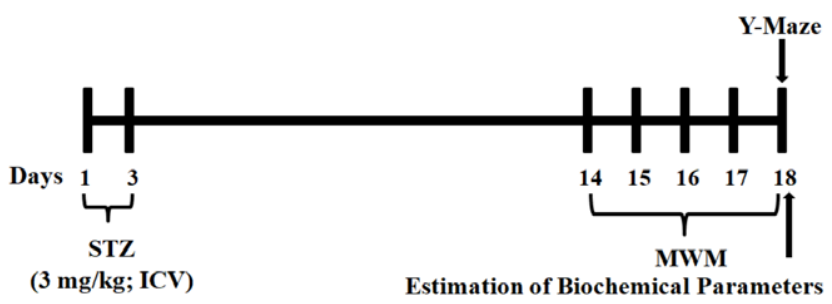

Figure 1: Diagrammatic representation of detailed experimental schedule. 
$\mathrm{MgCl}_{2} ; 1.7 \mathrm{mM} \mathrm{CaCl}_{2}$ and $2.2 \mathrm{mM}$ dextrose) was injected through Hamilton syringe using polyethylene tube into both side of lateral ventricle over a period of $5 \mathrm{~min}$ on day first followed by day third $(3 \mathrm{mg} / \mathrm{kg}$; $5 \mu \mathrm{L}$ each site at time lag of $5 \mathrm{~min}) \cdot{ }^{28,29} \mathrm{aCSF}(10 \mu \mathrm{l})$ was injected at a volume of $5 \mu \mathrm{l}$ into each site of the lateral ventricle of Sham animals. Quintessential Stereotaxic Injector (Stoelting, USA) was used for all microinjections.

\section{Assessment of STZ-induced Memory Impairment in Different Behavior Tests \\ Learning and Memory in Morris Water Maze Test Model}

Learning and memory of all the animals were quantified according to the MWM test procedure because it is one of the most widely used animal model to access memory and learning. ${ }^{30}$ Briefly, a circular black large pool of water tank (diameter $150 \mathrm{~cm}$; height $60 \mathrm{~cm}$ ) filled with water up to $30 \mathrm{CM}$ was included in the task to access learning and memory of animals. The water pool temperature was maintained at $26 \pm 1^{\circ} \mathrm{C}$. A water tank was partitioned into 4 equal quadrants with equal arc. A hidden platform (diameter $4.5 \mathrm{~cm}$ ) was placed below $1.5 \mathrm{~cm}$ of water surface in the center of target quadrant. Video tracking system was used to record the behavior of each animal. Randomly, each animal was placed in water tank for four consecutive trails (with the gap interval of $5 \mathrm{~min}$ between each trail) for $120 \mathrm{~s}$ in every day for four days from D-14 to D-17 of experimental protocol to search hidden platform and escape latency was recorded. On D-18, the hidden platform was taken out from the water pool during experimental procedure and tine spent in target quadrant and in each quadrant to search out platform was recorded. Swimming speed and percentage of total distance travelled in target quadrant were also recoded. Time spent in target quadrant was considered as marker of memory retrieval and memory building.

\section{Spontaneous Alteration Behavior in Y-Maze Test Paradigm}

The Y-maze test paradigm is considered to assess working memory of rodents. The spontaneous alteration behavior (SAB) was considered as index of working memory. Standard protocol was followed to observe SAB. ${ }^{31}$ Y-Maze (apparatus consist of three wooden arms labeled as A, B and C, $3 \mathrm{~cm}$ wide and $40 \mathrm{~cm}$ long with 12 $\mathrm{cm}$ of wall height) test paradigm was performed on lost day (D-18) of experimental protocol. Randomly each animal was placed at midpoint of T-maze apparatus for 8 min. During this time period rat was made free to move and successive entry sequence of $\mathrm{ABC}, \mathrm{BCA}$ or $\mathrm{CAB}$ (but not $\mathrm{ABA}$ ) and total number of arm entries were noted down. After each experiment the arms of Y-maze were wiped to remove resting odor.

\section{Evaluation of Effect of STZ on Cholinergic System}

\section{Sample Preparation}

HIP, PFC and AMY regions of brain were identified and homogenized in $01 \mathrm{~mL}$ of perchloric acid $(0.1 \mathrm{M})$ separately by a homogenizer. The homogenates were transferred into polypropylene tubes followed by addition of potassium acetate $(4 \mathrm{M} ; 50 \mu \mathrm{L})$ to maintain the $\mathrm{pH}$ to 4.0. Then resultant preparations were centrifuged at 4000Xg for $15 \mathrm{~min}^{32}$

\section{Assay of Choline Acetyltransferase (ChAT) Activity}

ChAT activity was analyzed spectrophotometrically $\left(\lambda_{\max }\right.$ $=450 \mathrm{~nm}$ ) with an enzyme-linked immunosorbent assay (ELISA) kit (SEB929Mu; Hubei, China) as directed by manufacturer and results were expressed as $\mathrm{n} \mathrm{mol} / \mathrm{hr} /$ mg protein.

\section{Quantification of ACh}

The level of $\mathrm{ACh}$ in discrete rat brain regions was quantified using Amplex red assay kit (Molecular Probes, inc., USA) as per standard protocol illustrated by Zoukhri and Kublin. ${ }^{33}$ In brief, homogenized tissue and $0.1 \mathrm{~mL}$ of $10 \mu \mathrm{M} \mathrm{H}_{2} \mathrm{O}_{2}$ (as control) were collected in three polypropylene tubes separately followed by addition of $0.1 \mathrm{~mL}$ of buffer $(50 \mathrm{mM}$ Tris-hydrochloric acid, $\mathrm{pH}$ 7.5) previously containing choline oxidase $(0.2$ $\mathrm{U} / \mathrm{mL}), \operatorname{AChE}(10 \mathrm{U} / \mathrm{mL})$, Amplex red reagent $(0.2 \mathrm{M})$ and horseradish peroxidase $(2 \mathrm{U} / \mathrm{mL})$ into each tubes. The fluorescence of each preparation was noted using spectrophotometer $\left(\lambda_{\max }=530 \mathrm{~nm}\right)$ at excitation $\left(\lambda_{\max }=\right.$ $530 \mathrm{~nm})$ and emission wavelength $\left(\lambda_{\max }=590 \mathrm{~nm}\right)$. The protein content for each brain regions was estimated as per set protocol. $^{34}$

\section{Estimation of AChE Activity in Discrete Brain Region of Rats}

To determine the AChE activity a standard protocol was followed as described in Amplex red assay kit (Molecular Probes, Inc., USA). Homogenized tissue was transferred in three separate tubes and $0.1 \mathrm{~mL}$ of buffer $(50 \mathrm{mM}$ Tris-hydrochloric acid, $\mathrm{pH}$ 7.5) containing Amplex red reagents $(400 \mu \mathrm{M})$, horseradish peroxidase $(2 \mathrm{U} /$ $\mathrm{mL})$, choline oxidase $(0.2 \mathrm{U} / \mathrm{mL})$ and $\mathrm{ACh}(100 \mu \mathrm{M})$ was added into each tube. After 30 min of incubation period, the fluorescence of each preparation was recorded using spectrophotometer at excitation $\left(\lambda_{\max }=530 \mathrm{~nm}\right)$ and emission wavelength $\left(\lambda_{\max }=590 \mathrm{~nm}\right)$. The amount of protein for each brain regions was estimated. ${ }^{34}$ 


\section{Quantification of Cytosolic A $\beta$ Level}

The A $\beta$ level was estimated with ELISA kit (Arigo Biolaboratories, Taiwan, ARG80939) using spectrophotometer $\left(\lambda_{\max }=450 \mathrm{~nm}\right)$ as per instructions of manufacturer.

\section{Evaluation of Effect of STZ on Mitochondrial Parameters in Discrete Brain Regions}

Mitochondrial Isolation from Discrete Rat Brain Tissues

Mitochondrial isolation from HIP, PFC and AMY was performed according to standard procedure illustrated by Pedersen. ${ }^{35}$ Thereafter, each tissue was implied for estimation of mitochondrial protein content using standard protocol of Lowry. ${ }^{34}$

\section{Assessment of Mitochondrial Function in Discrete Rat Brain Region}

Each rat brain tissue fraction was implied for evaluation of mitochondrial function by the method of reduction of 3-(4, 5-dimethylthiazol-2-yl)-2, 5 diphenyltetrazolium bromide (MT'T) using spectrophotometer $\left(\lambda_{\max }=595 \mathrm{~nm}\right){ }^{36}$ The level of Formosan formed was represented as $\mathrm{mg}$ formazan formed $/ \mathrm{min} / \mathrm{mg}$ protein.

\section{Estimation of Mitochondrial Membrane Potential (MMP) in Discrete Rat Brain Region}

MMP was evaluated using flurorescent cationic dye (tetramethyl rhodamine methylester; TMRM) to estimate the integrity of mitochondria. MMP estimation was performed using spectrophotometer at an excitation $\left(\lambda_{\max }=535 \pm 10 \mathrm{~nm}\right)$ and emission $\left(\lambda_{\max }=580 \pm 10 \mathrm{~nm}\right)$ as illustrated by Huang. ${ }^{37}$ The results were expressed as fluorescence intensity value/mg protein.

\section{Evaluation of Mitochondrial Bioenergetics in Discrete Rat Brain Region}

Polarography principle and Clark oxygen electrode (Hansatch Instruments Pvt. Ltd. USA) were used to evaluate extent of mitochondrial respiration. Respiratory control ratios (RCR) and $\mathrm{ADP} / \mathrm{O}$ were estimated as per the standard protocol. ${ }^{38}$

\section{Method of Protein Quantification}

A standard protocol was used to estimate protein concentration as explained by Bradford. ${ }^{39}$ To perform immunoblotting, discrete rat brain regions (HIP, PFC and AMY) were lysed separately in mixture of buffer and complete protease inhibitor cocktail. A standard curve of bovine serum albumin was plotted. For $\alpha 7$ nAChR protein, each aliquots of each sample were electrophoreses into 10\% SDS-PAGE gels and transferred to polyvinyllidene fluoride membranes and probed with specific antibody. Thereafter, membrane was incubated in phosphate buffered saline (PBS) buffer having milk $(5 \%)$ at room temperature for one h. After that membrane were probed with specific primary antibody (diluted with PBS, 2.5\% milk and $0.1 \%$ Twen 20) for overnight at $4^{\circ} \mathrm{C}$. Further membrane was incubated with rabbit anti- $\alpha 7 \mathrm{nAChR}$ (Abcam Plc., Cambridge, USA) primary polyclonal antibody (1:1000) for overnight. Thereafter, desired antibody was detected against $\alpha 7 \mathrm{nAChR}$ protein, then membrane was stripped with buffer (25 mM Glycine pH 2.0; $2 \%$ SDS) at room temperature for half an hours. Further, membrane was reprobed with anti- $\beta$-actin primary polyclonal antibody (1:500) to validate equal loading of protein for overnight. Thereafter, membrane was probed with analogous secondary antibody of $\beta$-actin. Immunoreactive band of protein was estimated with enhanced chemiluminescence (ECL) reagents by chemiluminescence. Thereafter, specific immunoreactive area was estimated by densitometric analysis with the help of Biovis gel documentation software.

\section{Evaluation of Oxidative Stress in Discrete Rat Brain Region}

\section{Assessment of Lipid Peroxidase Activity}

The lipid peroxidation (LPO) activity was evaluated calorimetrically $\left(\lambda_{\max }=535 \mathrm{~nm}\right)$ using standard protocol illustrated by Ohkawa. ${ }^{40}$ The LPO activity was measured in terms of malonaldialdehyde (MDA) concentration and represented in $\mu$ moles of $\mathrm{MDA} / \mathrm{mg}$ protein.

\section{Estimation of Level of Nitric Oxide (NO)}

The NO level was evaluated using standard method. ${ }^{41}$ The estimated result was represented in $\mathrm{n}$ moles of $\mathrm{NO} / \mathrm{mg}$ protein.

\section{Quantification of Catalase (CAT) Activity}

In the brain, CAT is responsible for breakdown of $\mathrm{H}_{2} \mathrm{O}_{2}$ which was estimated by spectrophotometry $\left(\lambda_{\max }=240 \mathrm{~nm}\right)$ using standard protocol. ${ }^{42}$ The resulted was represented as units $(\mathrm{U})$ of CAT activity/ $\mathrm{min} / \mathrm{mg}$ protein.

\section{Evaluation of Activity of Super Oxide Dismutase (SOD)}

The SOD activity was estimated with the help of spectrophotometer $\left(\lambda_{\max }=560 \mathrm{~nm}\right)$ using standard protocol. ${ }^{43}$ The assay is based on production of NADHphenazine methosulfate-nitro blue tetrazolium (NBT) formazan in discrete brain regions. The one unit $(\mathrm{U})$ of enzyme was represented as 50\% inhibition of NBT reduction $/ \mathrm{min} / \mathrm{mg}$ protein. 


\section{Data Analysis}

Values were presented as mean \pm standard error of mean (SEM). Repeated measures of two-way analysis of variance (ANOVA) followed by Bonferroni-post hoc test was performed for analysis of escape latency of rats on D-14 to D-17 in MWM test protocol. All other statistical analysis of obtained data was performed using one-way ANOVA followed by Student-NewmanKeuls post-hoc test to screen the significance between the groups. Value of $P<0.05$ was taken into consideration as significance.

\section{RESULTS}

\section{STZ Impairs Spatial Memory and Learning Ability of Rats in MWM Test Paradigm}

Figure 2 (A) illustrates the representative track plot of a rat of each group during MWM test. The effect of STZ on spatial and learning ability in terms of escape latency on D-14 to D-17 (B), time spent (C) and percentage of total distance travelled (D) in the target quadrant and swimming speed (E) on D-18 are depicted in Figure

(A)

\begin{tabular}{|c|c|c|c|c|c|}
\hline Groups & D-14 & D-15 & D-16 & D-17 & D-18 \\
\hline Control & & & & & \\
\hline Sham & & & & & \\
\hline sT2 & & & & & \\
\hline
\end{tabular}
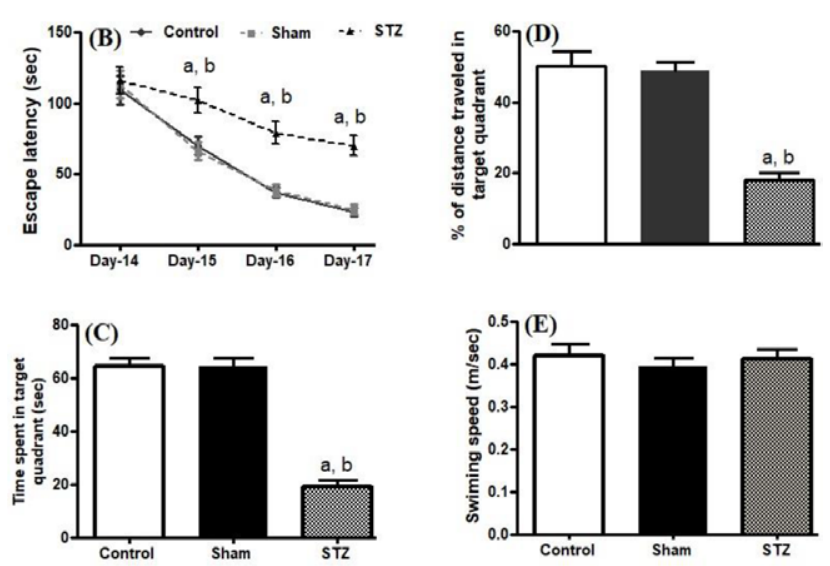

Figure 2: Representative tracking plot of an animal from all groups on D-14 to D-18 in Morris water maze (MWM) test paradigm (A). Effect of STZ on escape latency from D-14 to D-17 (B), time spent (C) and percentage of distance travelled (D) in the target quadrant and swimming speed of the animals (E) on D-18 during MWM test. All values are Mean \pm Standard Error of Mean (SEM, $n=6$ ). ${ }^{a} p<0.05$ compared to Control and ${ }^{\mathrm{b}} p<0.05$ compared to Sham (Two-way ANOVA followed by Bonferroni post-hoc test for escape latency from D-14 to D-17 and for other parameters one-way ANOVA followed by Student-Newman-Keuls post-hoc test).
2. Two-way ANOVA showed significant differences among group $\left(\mathrm{F}_{2,60}=26.16\right)$ and time $\left(\mathrm{F}_{3,60}=63.59\right)$ in the escape latency. Further, a significant interaction was observed in between group and time in the escape latency of the animals $\left(\mathrm{F}_{6,60}=2.32\right)$. post-hoc study revealed that there were no significant differences among groups in escape latency on D-14. STZ injection increased the escape latency of the animals on D-15 compared to other group rodents. These observations were similar on D-16 and D-17.

STZ administration significantly reduced the time spent $\left(\mathrm{F}_{2,15}=83.84\right)$ and percentage of distance travelled in target quadrant $\left(\mathrm{F}_{2,15}=38.63\right)$ on D-18 of MWM test than other group rats. Moreover, there were no significant differences in swimming speed of rats $\left(\mathrm{F}_{2,15}=0.3 .61\right)$ among the groups.

\section{STZ Impairs Spatial Working Memory Formation in Y-Maze Test}

Representative track plot of rat during Y-maze test was illustrated in Figure 3(A). Effect of STZ on change in spatial learning and memory in terms of percentage $S A B$ of animals is depicted in Figure 3(B). STZ significantly decreased the percentage of $\mathrm{SAB}$ during Y-maze test $\left(F_{2,15}=63.09\right)$ compared to other group rodents.

\section{STZ Impairs Cholinergic Activity in Rat HIP, PFC and $A M Y$}

Figure 4 illustrated the effect of STZ on cholinergic function in discrete region of brain in terms of activity

(A)

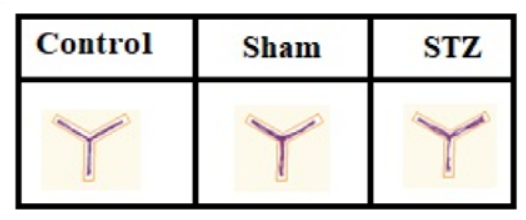

(B)

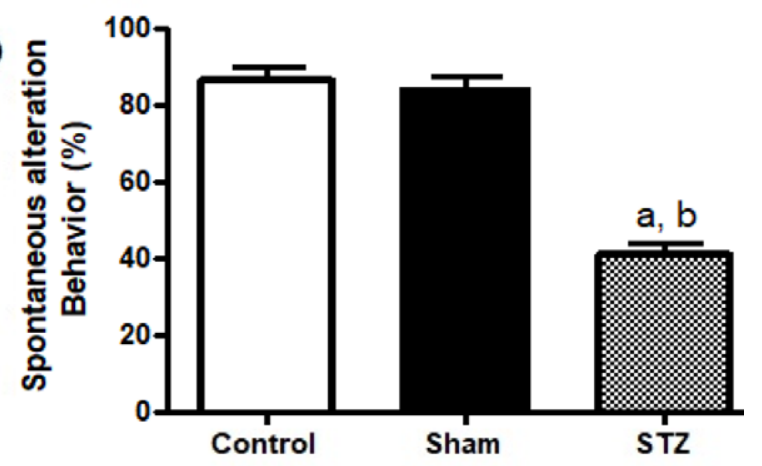

Figure 3: Representative tracking plot (A) of an animal from all groups on D-18 in Y-maze test paradigm; Effect of STZ on the percentage of spontaneous alteration behavior (SAB; B) of animals in Y-test paradigm. All values are Mean \pm SEM $(n=6)$. ${ }^{a} p<0.05$ compared to control and ${ }^{\mathrm{b}} p<0.05$ compared to Sham (One-way ANOVA followed by Student-Newman-Keuls post-hoc test). 
of ChAT (A), ACh level (B) and activity of AChE (C). STZ infusion significantly attenuated the activity of ChAT and the ACh level and increased the AChE activity in HIP $\left[\left(\mathrm{F}_{2,15}=32.5\right),\left(\mathrm{F}_{2,15}=40.90\right)\right.$ and $\left(\mathrm{F}_{2,15}=27.30\right)$ respectively], PFC $\left[\left(\mathrm{F}_{2,15} \stackrel{2,1}{=} 90.0\right),\left(\mathrm{F}_{2,15}=37.50\right)\right.$ and $\left(\mathrm{F}_{2,15}\right.$ $=19.7)$ respectively $]$ and $\operatorname{AMY}\left[\left(\mathrm{F}_{2,15}=122.7\right),\left(\mathrm{F}_{2,15}=\right.\right.$ $20.0)$ and $\left(F_{2,15}=20.20\right)$ respectively] compared to other group animals.

\section{STZ Increases the Level of Amyloid Beta in Rat Brain Regions}

Effect of STZ on A $\beta$ level is illustrated in Figure 5. STZ infusion significantly increased the level of $A \beta$ in HIP

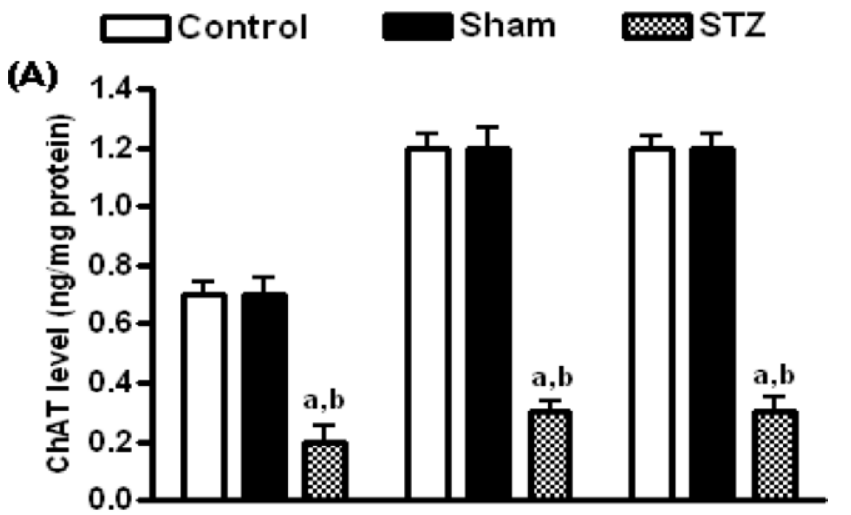

(B)
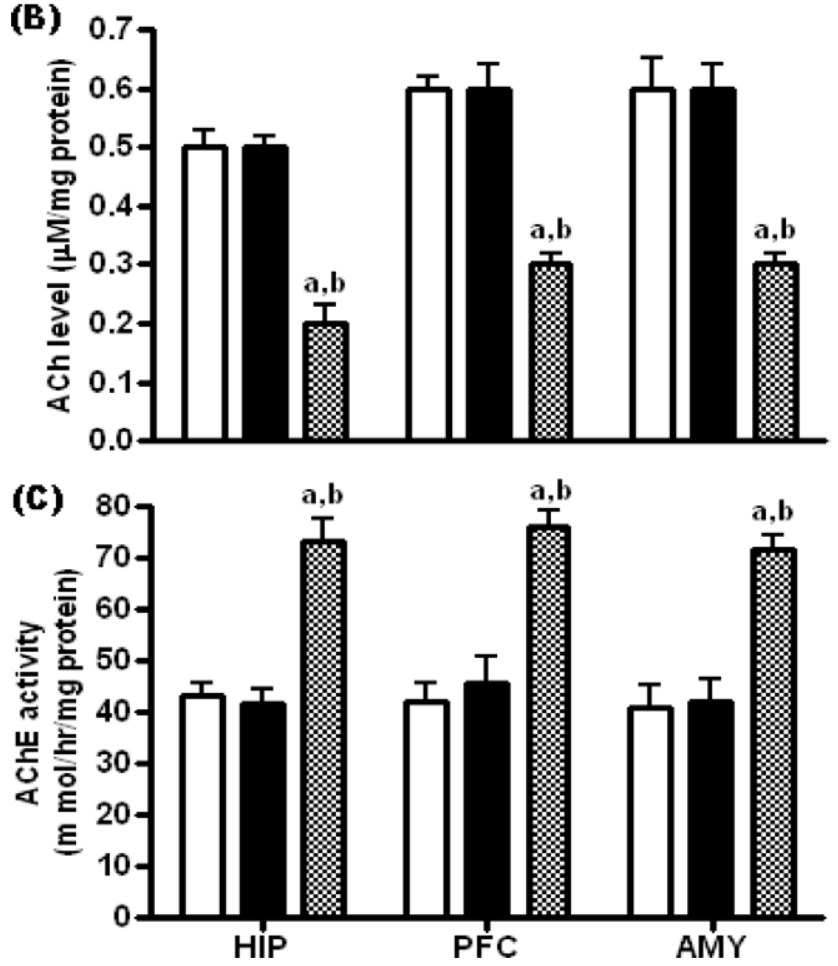

Figure 4: Effect of STZ on change in the level of choline acetyltransferase (ChAT; A) and acetylcholine (ACh; B) and activity of acetylcholinesterase (AChE; C) in the rat hippocampus (HIP), pre-frontal cortex (PFC) and amygdala (AMY). All values are Mean $\pm \operatorname{SEM}(n=6)$. ${ }^{a} p<0.05$ compared to control and ${ }^{b} p<0.05$ compared to Sham (One-way ANOVA followed by Student-Newman-Keuls post-hoc test).
$\left(F_{2,15}=91.6\right), \operatorname{PFC}\left(F_{2,15}=49.4\right), \operatorname{AMY}\left(F_{2,15}=99.9\right)$ than other two group rats.

\section{STZ Reduces the Expression Level of $\alpha 7$ nAChR in Discrete Rat Brain Regions}

Figure 6 illustrated the effect of STZ on the expression level of $\alpha 7 \mathrm{nAChR}$ in selected rat brain regions. STZ infusion significantly attenuated the $\alpha 7 \mathrm{nAChR}$ level in $\operatorname{HIP}\left(\mathrm{F}_{2,15}=59.5\right)$, PFC $\left(\mathrm{F}_{2,15}=54.9\right)$ and $\mathrm{AMY}$ $\left(\mathrm{F}_{2,15}=76.8\right)$ compared to other two group rats.

STZ Attenuates Function and Integrity of Mitochondria in Rat Brain Regions

Figure 7 illustrated the effect of STZ on the function in the terms of the level of formazan produced in MTT assay (A) and integrity in terms of the fluorescence intensity of TMRM (B) of mitochondria in memorysensitized rat brain regions. STZ infusion significantly decreased the function and integrity of mitochondria in $\mathrm{HIP}\left[\left(\mathrm{F}_{2,15}=11.0\right)\right.$ and $\left(\mathrm{F}_{2,15}=62.2\right)$ respectively $]$, PFC $\left[\left(\mathrm{F}_{2,15}=10.5\right)\right.$ and $\left(\mathrm{F}_{2,15}=56.7\right)$ respectively $]$ and AMY $\left[\left(\mathrm{F}_{2,15}=8.1\right)\right.$ and $\left(\mathrm{F}_{2,15}=54.2\right)$ respectively $]$ compared to other two group rodents.

\section{STZ Decreases Mitochondrial Bioenergetics in Rat Brain Regions}

Effect of STZ on the alteration in the level of $\mathrm{O}_{2}$ utilization in different stages of Oxygraph in rat HIP (A), PFC (B) and AMY (C) is depicted in Figure 8. The effect of STZ on the mitochondrial RCR (A) and $\mathrm{ADP} / \mathrm{O}(\mathrm{B})$ is illustrated in Figure 9. STZ infusion significantly decreased RCR and ADP/O in rat HIP $\left[\left(\mathrm{F}_{2,15}=19.4\right)\right.$ and $\left(\mathrm{F}_{2,15}=96.0\right)$ respectively $]$, PFC $\left[\left(\mathrm{F}_{2,15}\right.\right.$ $=20.1)$ and $\left(\mathrm{F}_{2,15}=27.3\right)$ respectively $]$ and $\mathrm{AMY}\left[\left(\mathrm{F}_{2,15}\right.\right.$

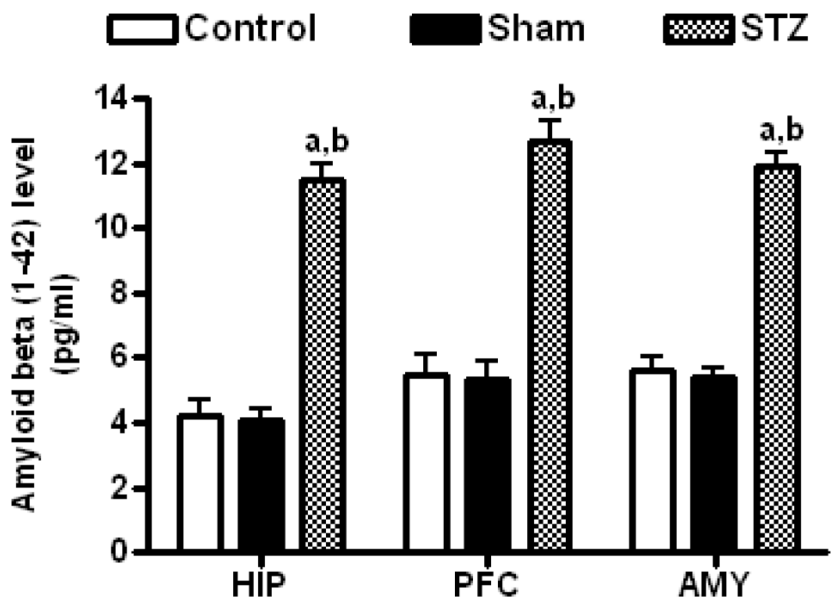

Figure 5: Effect of STZ on the level of amyloid beta (A $\beta)$ in the rat HIP, PFC and AMY. All values are Mean \pm SEM $(n=6)$. ${ }^{a} p<0.05$ compared to Control and ${ }^{b} p<0.05$ compared to Sham

(One-way ANOVA followed by Student-Newman-Keuls post-hoc test). 


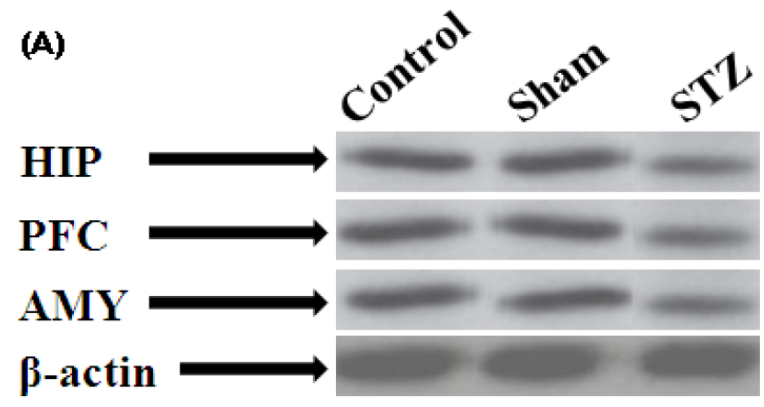

(B) $\square$ Control $\square$ Sham $\lll S T Z$

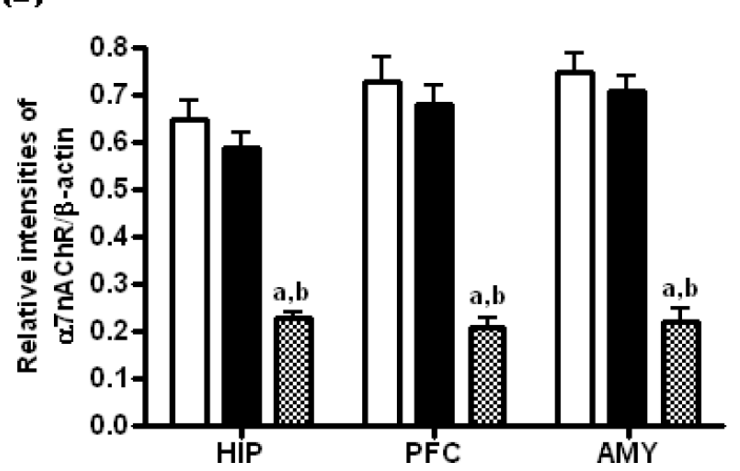

Figure 6: Effect of STZ on the level of expression of $\alpha 7$ nACh receptor in the rat HIP, PFC and AMY. All values are Mean \pm SEM $(n=6) .{ }^{a} p<0.05$ compared to control and ${ }^{b} p<0.05$ compared to Sham (One-way ANOVA followed by StudentNewman-Keuls Post-hoc test).
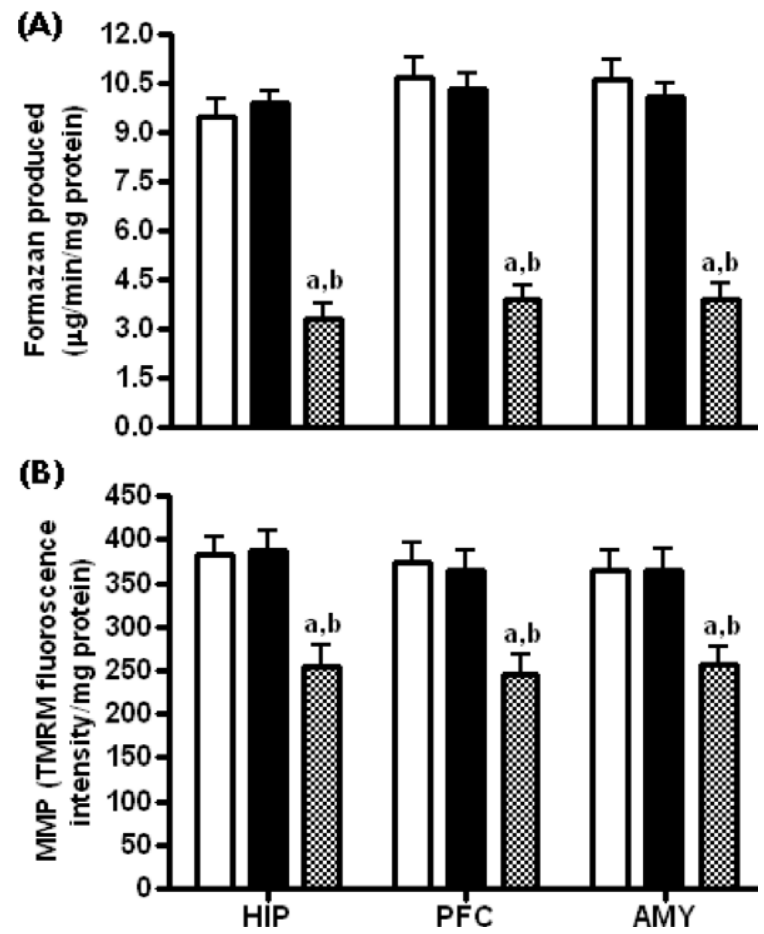

Figure 7: Effect of STZ on the mitochondrial function in terms of level of formazan produced in MTT assay (A) and integrity in terms of fluorescence intensity of TMRM $(B)$ in the rat HIP, PFC and AMY. All values are Mean $\pm \operatorname{SEM}(n=6) .{ }^{\mathrm{a}} p<0.05$ compared to control and ${ }^{\mathrm{b}} p<0.05$ compared to Sham (Oneway ANOVA followed by Student-Newman-Keuls post-hoc test).
$=24.4)$ and $\left(\mathrm{F}_{2,15}=92.2\right)$ respectively] compared to other two group animals.

\section{STZ Increases Markers of Oxidative and Nitrosative Stress in the Rat Brain Regions}

Effect of STZ on mitochondrial level of NO and LPO and activity of CAT and SOD in rat HIP, PFC and AMY is illustrated in Table 1. STZ infusion significantly
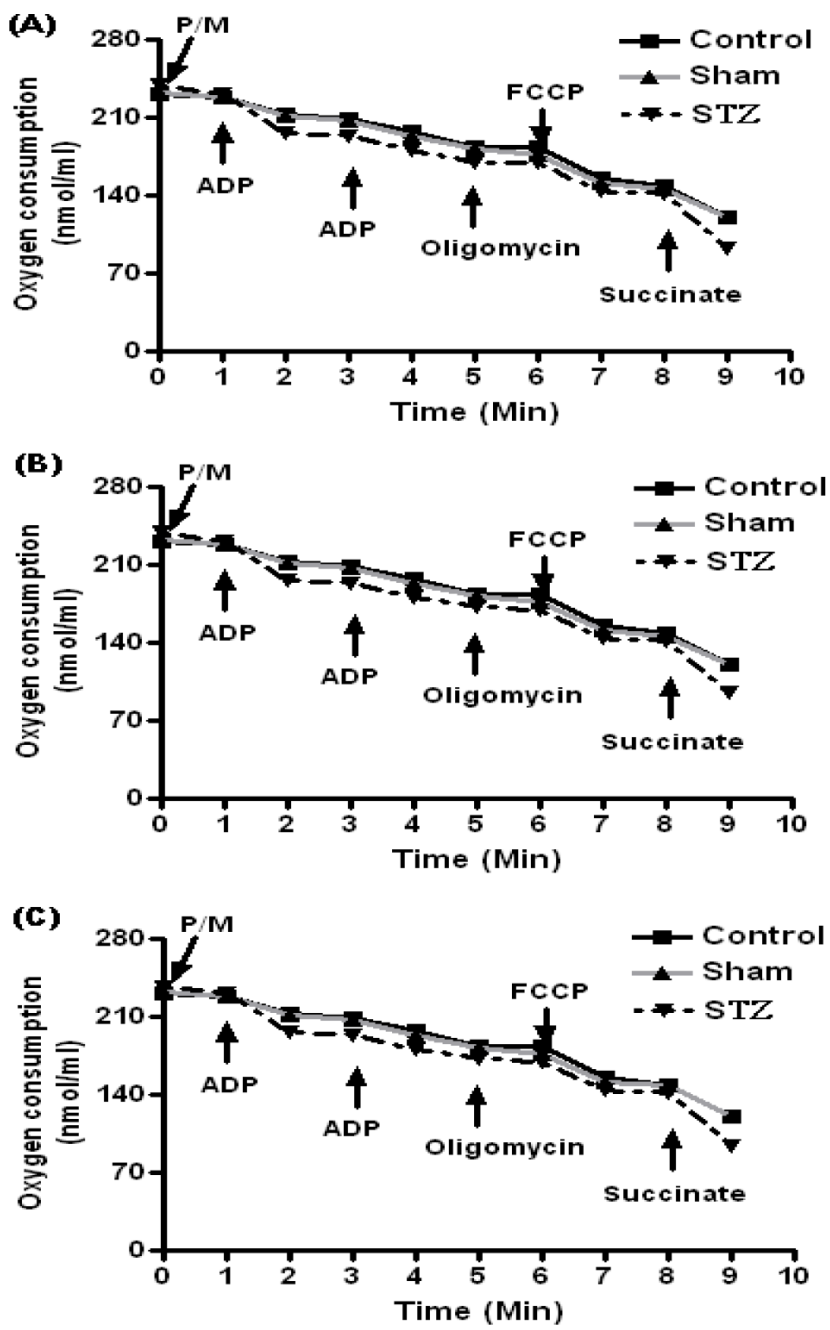

Figure 8: Representative Oxygraph of HIP (A), PFC (B) and AMY (C) of an animal in each group of the experimental protocol to show the level of oxygen consumption in different stages of mitochondrial respiration. Briefly, at first the initial rate of oxygen consumption (state 2 orV2) was recorded after addition of pyruvate plus malate $(10 \mathrm{mM} / 5 \mathrm{mM})$. Further,

the state 3 rate of oxygen consumption was recorded after addition of ADP $(250 \mathrm{nmol})$. Moreover, a measurable state 4 rate of oxygen consumption (i.e., the rate after ADP phosphorylation) was monitored when a second pulse of ADP was added but the phosphorylative cycle was soon inhibited before its completion by adding oligomycin $(1 \mu \mathrm{g})$. Subsequently, a measurable oligomycin oxygen consumption rate was recorded after FCCP $(1 \mu \mathrm{M})$ was added to obtain a rate of oxygen consumption in the absence of coupled oxidative phosphorylation. Further, the oxygen consumption was recorded in presence of succinate $(15 \mathrm{mM})$ and rotenone (2.2 $\mathrm{mM}$ ) to observe the respiratory complex-II activity. 
(A) $\square$ Control $\square$ Sham
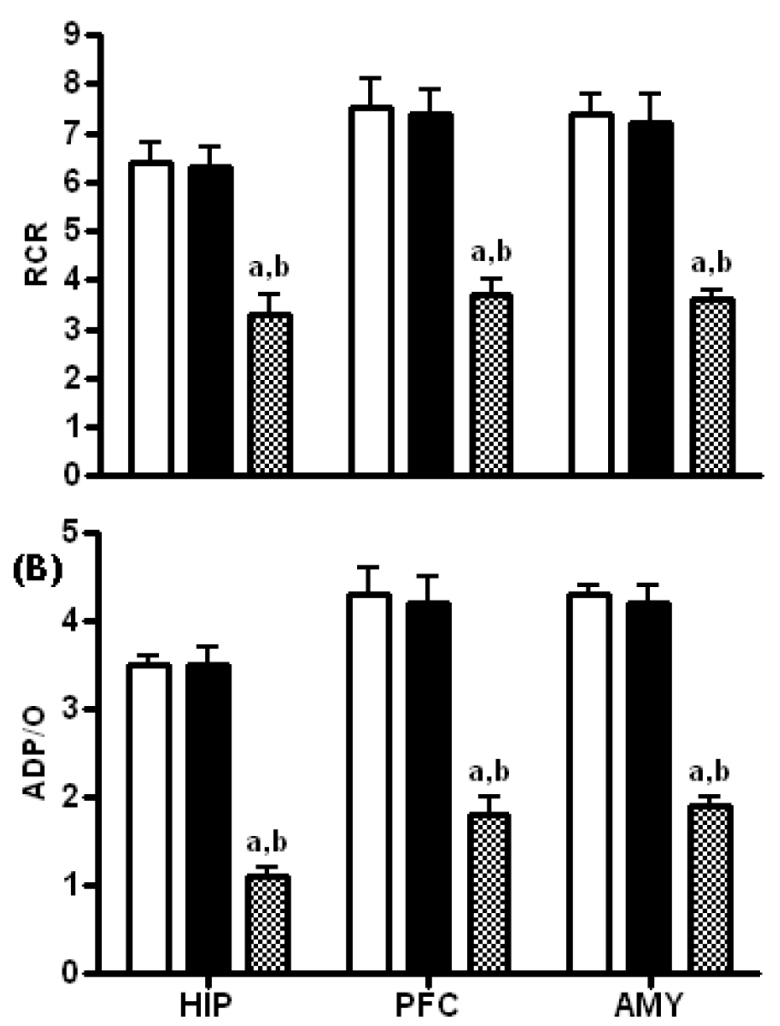

Figure 9: Effect of STZ on the mitochondrial respiratory control ratio (RCR; A) and ADP/O (B) in rat HIP, PFC and AMY. All values are Mean \pm SEM $(n=6)$. ${ }^{a} p<0.05$ compared to Control and ${ }^{b} p<0.05$ compared to Sham (One-way ANOVA followed by Student-Newman-Keuls post-hoc test).

increased the mitochondrial $\mathrm{NO}$ and LPO levels and decreased CAT and SOD activity in HIP $\left[\left(\mathrm{F}_{2,15}=48.5\right)\right.$, $\left(\mathrm{F}_{2,15}=50.0,\left(\mathrm{~F}_{2,15}=15.6\right)\right.$ and $\left(\mathrm{F}_{2,15}=63.4\right)$ respectively $]$, PFC $\left[\left(\mathrm{F}_{2,15}=97.5\right),\left(\mathrm{F}_{2,15}=55.5\right),\left(\mathrm{F}_{2,15}=24.9\right)\right.$ and $\left(\mathrm{F}_{2,15}\right.$ $=17.9)$ respectively $]$ and $\operatorname{AMY}\left[\left(\mathrm{F}_{2,15}=26.0\right),\left(\mathrm{F}_{2,15}\right.\right.$ = 26.2), $\left(\mathrm{F}_{2,15}=18.4\right)$ and $\left(\mathrm{F}_{2,15}=38.5\right)$ respectively] compared to other two group rats.

\section{DISCUSSION}

We for the first time report a reduction in the $\alpha 7 \mathrm{nAChR}$ level in the brain regions of the STZ-challenged animals. Further, ICV injection of STZ impairs function, integrity and bioenergetics of mitochondria in the rat brain regions. Results also indicate a remarkable reduction in cholinergic function and elevation in the $A \beta$ level in the above rat brain regions. Thus, there may be a relationship between the level of expression of alpha7 $\mathrm{nAChR}$ and function of mitochondria in the STZ-challenged brain areas of the rats.

The present study also suggests that ICV injection of STZ significantly reduced the learning and memory during MWM test paradigm and decreased the spatial
Table 1: Effect of STZ on mitochondrial level of

NO and LPO and activity of SOD and CAT in rat HIP, PFC and AMY.

\begin{tabular}{|c|c|c|c|}
\hline Tissue & Control & Sham & STZ \\
\hline \multicolumn{4}{|c|}{ NO ( $\mu$ M MDA/mg protein) } \\
\hline HIP & $1.1 \pm 0.03$ & $1.2 \pm 0.12$ & $2.3 \pm 0.11^{\text {a,b }}$ \\
\hline PFC & $1.3 \pm 0.02$ & $1.2 \pm 0.06$ & $2.5 \pm 0.11^{\text {a,b }}$ \\
\hline AMY & $1.3 \pm 0.03$ & $1.3 \pm 0.04$ & $2.4 \pm 0.21^{\text {a,b }}$ \\
\hline \multicolumn{4}{|c|}{ LPO (nmol of MDA/mg of protein) } \\
\hline HIP & $0.6 \pm 0.01$ & $0.5 \pm 0.03$ & $0.9 \pm 0.04^{\text {a,b }}$ \\
\hline PFC & $0.9 \pm 0.02$ & $1.0 \pm 0.04$ & $1.9 \pm 0.12^{\text {a,b }}$ \\
\hline AMY & $0.9 \pm 0.13$ & $0.8 \pm 0.13$ & $1.8 \pm 0.03^{\text {a,b }}$ \\
\hline \multicolumn{4}{|c|}{ SOD (Units/min/mg of protein) } \\
\hline HIP & $0.5 \pm 0.02$ & $0.5 \pm 0.03$ & $0.2 \pm 0.01^{\text {a,b }}$ \\
\hline PFC & $0.7 \pm 0.07$ & $0.8 \pm 0.08$ & $0.3 \pm 0.02^{\text {a,b }}$ \\
\hline AMY & $0.8 \pm 0.05$ & $0.8 \pm 0.06$ & $0.3 \pm 0.02^{\text {a,b }}$ \\
\hline \multicolumn{5}{|c|}{ CAT (Units/min/mg of protein) } \\
\hline HIP & $2.1 \pm 0.11$ & $2.1 \pm 0.13$ & $1.3 \pm 0.11^{\text {a,b }}$ \\
\hline PFC & $2.7 \pm 0.03$ & $2.6 \pm 0.10$ & $1.7 \pm 0.16^{\text {a,b }}$ \\
\hline AMY & $2.7 \pm 0.19$ & $2.7 \pm 0.14$ & $1.6 \pm 0.10^{\text {a,b }}$ \\
\hline
\end{tabular}

All values are mean \pm SEM $(n=6)$. ${ }^{a} p<0.05$ compared to Control and ${ }^{b} p<0.05$ compared to Sham (one-way ANOVA followed by Student-Newman-Keuls test).

memory during Y-maze test as reported previously. ${ }^{23}$ STZ treatment showed significant decrease in the learning ability of the rats in this protocol throughout the study (D-14 to D-17). Further, STZ exhibited significant decrease in the percentage of total distance travelled and time spent in the target quadrant in the probe session, indicating that STZ attenuated memory formation. Additionally, STZ significantly attenuated spatial memory in terms of percentage of SAB during Y-maze paradigm, suggesting the fact that STZ reduces the short-term memory formation. It is well established that the AD leads to decline in MWM task performance in rodents and MWM learning and memory status provide a good approach for screening of agents with anti-AD potential. ${ }^{44}$ In addition to this, ICV injection of STZ also increased A $\beta$ level in the above all three brain regions as appears in AD. Our findings show that the cholinergic dysfunction associated with alterations in mitochondrial bioenergetics and oxidative status could be considered as early events in pathophysiology of AD. ICV-STZ administration can induce various physiological, pathological and behavioral changes in animals including memory impairment, metabolic dysfunctions, oxidative stress and many more. ${ }^{45}$ STZ has been shown to enhance AChE activity in cognitive deficits sensitized rodent brain. ${ }^{46,47}$ In the present study, AChE activity was significantly elevated in STZ-challenged rat brain which is similar to that of Sonkusare et al. ${ }^{48}$ ICV-STZ injection 
in rats leads to progressive deficits in memory and learning and also develops various pathological aspects similar to $\mathrm{AD}{ }^{25}$ In addition to increase in the $\mathrm{AChE}$ activity, ChAT performance also diminishes in STZchallenged rodents. Thus, cholinergic dysfunction also leads to diminish the rate of ACh synthesis in memorysensitized rat brain regions. ${ }^{49,50}$ It is well reported that excessive reactive oxygen species (ROS) generation in neuronal cell also contribute in the phathogenesis of AD. ${ }^{51,52}$ Mitochondria are the primary source of energy and produce about $90-95 \%$ cellular ATP by oxidative phosphylation. The mitochondrial ETC and NADPH oxidase are considered as the most important source for cellular ROS. Mitochondrial dysfunction leads to reduced ATP production and results into energy metabolic crises. ${ }^{53,54}$ impaired energy metabolisms has been reported in $\mathrm{AD}$-like brain. Increased oxidative stress derived from mitochondrial dysfunction facilitates the neurodegeneration. ${ }^{45}$ Further, ICV-STZ injection altered bioenergetics and mitochondrial integrity that leads to the inhibition of ATP synthesis in memorysensitized brain rat which is similar to pervious finding. ${ }^{47}$ Thus, ICV-STZ administration induced oxidative stress dependent mitochondrial dysfunction and it may be postulated that energy impairment caused by STZ may be a potential source for this oxidative stress.

Loss or down regulation of the neuronal nAChRs is also reported in $\mathrm{AD}$ pathophysiology. ${ }^{55}$ We also found that ICV-STZ administration altered the expression of $\alpha 7 \mathrm{nAChR}$ in memory-sensitized rat brain which led to persistent impairment in learning and memory and quantified in terms of decrease expression of $\alpha 7 \mathrm{nAChR}$ in above brain regions of rats. The nAChRs especially $\alpha 7 \mathrm{nAChRs}$ are well expressed in the neuron of brain and mainly in HIP, PFC and AMY. It has been well accepted that $\alpha 7 \mathrm{nAChRs}$ activation is a useful therapeutic approaches for management of $\mathrm{AD}$ because this receptor maintains the cholinergic phenotype. ${ }^{25,56}$ Thus, it can be assumed that there may be a significant role of $\alpha 7 \mathrm{nAChRs}$ in the STZ-challenged memorysensitized brain regions of cognitive impaired rats which is a matter of future experiments.

\section{CONCLUSION}

In conclusion, STZ treatment attenuates $\alpha 7 \mathrm{nAChR}$ expression and mitochondrial dysfunctions in all three memory-ensitized rat brain regions. Further, the cholinergic functions are reduced in STZ treated rat brain and level of $A \beta$ is increased in all three brain regions of such animals. Therefore, results indicate that there could be a strong relationship between $\alpha 7 \mathrm{nAChR}$ expression and dysfunction in mitochondria in memorysensitive areas of STZ-challenged animals. Thus, it can be speculated that $\alpha 7 \mathrm{nAChR}$-dependent mitochondrial function could be an alternate target to enhance memory formation in $\mathrm{AD}$.

\section{ACKNOWLEDGEMENT}

NKS is thankful to GLA University, Mathura and Uttar Pradesh, India for the financial support.

\section{CONFLICT OF INTEREST}

The authors declare that they have no conflict of interests.

\section{ABBREVIATIONS}

AD: Alzheimer's disease; nAChRs: Nicotinic Acetylcholine Receptors; A $\beta$ : Amyloid Beta; ACh: Acetylcholine; ROS: Reactive Oxygen Species; ICV: Intracerebroventricular; STZ: Streptozotocin; aCSF: Artificial Cerebrospinal Fluid; IEAC: Institutional Animal Ethics Committee; CPCSEA: Committee for the Purpose of Control and Supervision of Experiments in Animals' AChE: Acetylcholinesterase; ChAT: Choline Acetyltransferase; MWM: Morris Water Maze; SAB: spontaneous alteration behavior; HIP: Hippocampus; PFC: Prefrontal Cortex; AMY: Amygdale; ANOVA: Analysis of Variance; MTT: 3-(4, 5-Dimethylthiazol-2-yl)-2, 5 Diphenyltetrazolium Bromide; TMRM: Tetramethyl Rhodamine Methylester; MMP: Mitochondrial Membrane Potential; RCR: Respiratory Control Ratio; LPO: Lipid Peroxidation; MDA: Malonaldialdehyde; CAT: Catalase; SOD: Super Oxide Dismutase.

\section{REFERENCES}

1. Shen J, Wu J. Nicotinic cholinergic mechanisms in alzheimer's disease. Int Rev Neurobiol. 2015;124:275-92.

2. Sadigh-Eteghad S, Majdi A, Talebi M, Mahmoudi J, Babri S. Regulation of nicotinic acetylcholine receptors in Alzheimer's disease: A possible role of chaperones. Eur J Pharmacol. 2015;755:34-41.

3. World Health Organization and Alzheimer's Diseaselnternational. Dementia: A public health priority. 2012;112. Available from: http://www.who.int/mental_ health/publications/dementia_report_2012/en/.

4. Aggarwal NT, Shah RC, Bennett DA. Alzheimer's disease: Unique markers for diagnosis and new treatment modalities. Indian $\mathrm{J}$ Med Res. 2015;142(4):369-82.

5. Yiannopoulou KG, Papageorgiou SG. Current and future treatments for Alzheimer's disease. Ther Adv Neurol Disord. 2013;6(1):19-33.

6. Utsugisawa K, Nagane Y, Tohgi H, Yoshimura M, Ohba H, Genda Y. Changes with aging and ischemia in nicotinic acetylcholine receptor subunit $\alpha 7 \mathrm{mRNA}$ expression in postmortem human frontal cortex and putamen. Neurosci Lett. 1999;270(3):145-8.

7. Nordberg A. Nicotinic receptor abnormalities of Alzheimer's disease: Therapeutic implications. Biol Psychiatry. 2001;49(3):200-10. 
8. Wu J, Kuo YP, George AA, Xu L, Hu J, Lukas RJ. $\beta$-amyloid directly inhibits human $\alpha 4 \beta 3$-nicotinic acetylcholine receptors heterologously expressed in human SH-EP1 cells. J Biol Chem. 2004;279(36):37842-51.

9. Liu Q, Huang Y, Xue F, Simard A, DeChon J, Li GG, et al. A novel nicotinic acetylcholine receptor subtype in basal forebrain cholinergic neurons with high sensitivity to amyloid peptides. J Neurosci. 2009;29(4):918-29.

10. Pettit DL, Shao Z, Yakel JL. Beta-Amyloid(1-42) peptide directly modulates nicotinic receptors in the rat hippocampal slice. J Neurosci. 2001;21(1):1-5.

11. Lykhmus O, Voytenko L, Koval L, Mykhalskiy S, Kholin V, Peschana K, et al. $\alpha 7$ nicotinic acetylcholine receptor-specific antibody induces inflammation and amyloid $\beta 42$ accumulation in the mouse brain to impair memory. PLoS One. 2015;10(3):1-18.

12. Lykhmus O, Gergalova G, Zouridakis M, Tzartos S, Komisarenko S, Skok M. Inflammation decreases the level of alpha7 nicotinic acetylcholine receptors in the brain mitochondria and makes them more susceptible to apoptosis induction. Int Immunopharmacol. 2015;29(1):148-51.

13. Liu Q, Zhang J, Zhu H, Qin C, Chen Q, Zhao B. Dissecting the signaling pathway of nicotine-mediated neuroprotection in a mouse Alzheimer disease model. FASEB J. 2007;21(1):61-73.

14. Lahiri DK, Utsuki T, Chen D, Farlow MR, Shoaib M, Ingram DK. et al. Nicotine reduces the secretion of Alzheimer's beta-amyloid precursor protein containing beta-amyloid peptide in the rat without altering synaptic proteins. Ann N Y Acad Sci. 2002;965(1):364-72.

15. Fuenzalida M, Pérez MA, Arias HR. Role of nicotinic and muscarinic receptors on synaptic plasticity and neurological diseases. Curr Pharm Des. 2016;22(14):2004-14.

16. Bitner RS, Nikkel AL, Markosyan S, Otte S, Puttfarcken P, Gopalakrishnan $M$. Selective $\alpha 7$ nicotinic acetylcholine receptor activation regulates glycogen synthase kinase $3 \beta$ and decreases tau phosphorylation in vivo. Brain Res. 2009;1265:65-74.

17. Sadot E, Gurwitz D, Barg J, Behar L, Ginzburg I, Fisher A. Activation of $\mathrm{m} 1$ muscarinic acetylcholine receptor regulates tau phosphorylation in transfected $P_{12}$ cells. J Neurochem. 2002;66(2):877-80.

18. Gergalova G, Lykhmus O, Kalashnyk O, Koval L, Chernyshov V, Kryukova E, et al. Mitochondria express $\alpha 7$ nicotinic acetylcholine receptors to regulate $\mathrm{Ca}^{2+}$ accumulation and cytochrome $\mathrm{c}$ release: Study on isolated mitochondria. PLoS One. 2012;7(2):2-9.

19. Drisdel RC, Green WN. Neuronal $\alpha$-bungarotoxin receptors are $\alpha 7$ subunit homomers. J Neurosci. 2000;20(1):133-9.

20. Sharma M, Gupta YK. Intracerebroventricular injection of streptozotocin in rats produces both oxidative stress in the brain and cognitive impairment. Life Sci. 2001;68(9):1021-9.

21. Grunblatt E, Salkovic-Petrisic M, Osmanovic J, Riederer P, Hoyer S. Brain insulin system dysfunction in streptozotocin intracerebroventricularly treated rats generates hyperphosphorylated tau protein. J Neurochem. 2007;101(3):757-70.

22. Correia SC, Santos RX, Santos MS, Casadesus G, Lamann JC, Perry G, et al. Mitochondrial abnormalities in a streptozotocin-induced rat model of sporadic alzheimer disease. Curr Alzheimer Res. 2013;10(4):406-19.

23. Saxena G, Patro IK, Nath C. ICV STZ induced impairment in memory and neuronal mitochondrial function: A protective role of nicotinic receptor. Behav Brain Res. 2011;224(1):50-7.

24. Esteves IM, Lopes-Aguiar C, Rossignoli MT, Ruggiero RN, Broggini ACS, Bueno-Junior LS, et al. Chronic nicotine attenuates behavioral and synaptic plasticity impairments in a streptozotocin model of Alzheimer's disease. Neuroscience. 2017;353:87-97.

25. Tota S, Kamat PK, Shukla R, Nath C. Improvement of brain energy metabolism and cholinergic functions contributes to the beneficial effects of silibinin against streptozotocin induced memory impairment. Behav Brain Res. 2011;221(1):207-15.

26. Paxinos G, Watson C. The Rat Brain in Stereotaxic Coordinates. Acad Press, New York. 1998;4.

27. Palkovits M, Brownstein MJ. Maps and guide to micro dissection of the rat brain. Elsevier, New York, NY, USA. 1988.

28. Agrawal R, Mishra B, Tyagi E, Nath C, Shukla R. Effect of curcumin on brain insulin receptors and memory functions in STZ (ICV) induced dementia model of rat. Pharmacol Res. 2010;61(3):247-52.
29. Kumar M, Bansal N. Fasudil hydrochloride ameliorates memory deficits in rat model of streptozotocin-induced Alzheimer's disease: Involvement of PI3kinase, eNOS and NFKB. Behav Brain Res. 2018;351:4-16.

30. Morris R. Developments of a water-maze procedure for studying spatial learning in the rat. J Neurosci Methods. 1984;11(1):47-60.

31. Mouri A, Noda $Y$, Hara $H$, Mizoguchi $H$, Tabira $T$, Nabeshima $T$. Oral vaccination with a viral vector containing $A \beta C D N A$ attenuates age-related $A \beta$ accumulation and memory deficits without causing inflammation in a mouse Alzheimer model. FASEB J. 2007;21(9):2135-48.

32. Muthuraju S, Maiti P, Solanki P, Sharma AK, Singh SBA, et al. Acetylcholinesterase inhibitors enhance cognitive functions in rats following hypobaric hypoxia. Behav Brain Res. 2009;203(1):1-14.

33. Zoukhri $\mathrm{D}$, Kublin $\mathrm{CL}$. Impaired neurotransmitter release from lacrimal and salivary gland nerves of a murine model of sjögren's syndrome. Investig Ophthalmol Vis Sci. 2001;42(5):925-32.

34. Lowry OH, Rosenborough NJ, Farr AL, Randall RJ. Protein measurement with folin phenol reagent. J Biol Chem. 1951;193:265-75.

35. Pedersen PL, Greenawalt JW, Reynafarje B, Hullihen J, Decker GL, Soper JW, et al. Preparation and characterization of mitochondriaand submitochondrial particles of rat liver and liver-derived tissues. Methods Cell Biol. 1978;20:411-81.

36. Kamboj SS, Kumar V, Kamboj A, Sandhir R. Mitochondrial oxidative stress and dysfunction in rat brain induced by carbofuran exposure. Cell Mol Neurobiol. 2008;28(7):961-9.

37. Huang SG. Development of a high throughput screening assay for mitochondrial membrane potential in living cells. J Biomol Screen. 2002;7(4):383-9.

38. Chance B, Williams G. Respiratory enzymes in oxidative phosphorylation. J Biol Chem. 1956;217(1):409-28.

39. Bradford MM. A rapid and sensitive method for the quantitation of microgram quantities of protein utilizing the principle of protein-dye binding. Anal Biochem. 1976;72(1-2):248-54.

40. Ohkawa H, Ohishi N, Yagi K. Assay for lipid peroxides in animal tissues by thiobarbituric acid reaction. Anal Biochem. 1979;95(2):351-8.

41. Green LC, Wagner DA, Glogowski J, Skipper PL, Wishnok JS, Tannenbaum $\mathrm{SR}$. Analysis of nitrate, nitrite and $[15 \mathrm{~N}]$ nitrate in biological fluids. Anal Biochem. 1982;126(1):131-8.

42. Beers RF, Sizer IW. A spectrophotometric method for measuring the breakdown of hydrogen peroxide by catalase. J Biol Chem. 1952;195(1):133-40.

43. Kakkar P, Das B, Viswanathan PN. A modified spectrophotometric assay of superoxide dismutase. Indian J Biochem Biophys. 1984;21(2):130-2.

44. Vorhees CV, Williams MT. Morris water maze: Procedures for assessing spatial and related forms of learning and memory. Neurology. 2006;1(2):848-58.

45. Li L, Qin L, Lu HL, Li PJ, Song YJ, Yang RL. Methylene blue improves streptozotocin-induced memory deficit by restoring mitochondrial function in rats. Brain Res. 2017;1657:208-14.

46. Maratha SR, Mahadevan N. Memory enhancing activity of naringin in unstressed and stressed mice: Possible cholinergic and nitriergic modulation. Neurochemical Research. 2012;37(10)2206-12.

47. Sachdeva AK, Kuhad A, Chopra K. Naringin ameliorates memory deficits in experimental paradigm of Alzheimer's disease by attenuating mitochondrial dysfunction. Pharmacol Biochem Behav. 2014;127:101-10.

48. Sonkusare S, Srinivasan K, Kaul C, Ramarao P. Effect of donepezil and lercanidipine on memory impairment induced by intracerebroventricular streptozotocin in rats. Life Sci. 2005;77(1):1-14.

49. Tota S, Kamat PK, Awasthi H, Singh N, Raghubir R, Nath $\mathrm{C}$, et al. Candesartan improves memory decline in mice: Involvement of AT1 receptors in memory deficit induced by intracerebral streptozotocin. Behav Brain Res. 2009;199(2):235-40.

50. Hellweg R, Nitsch R, Hock C, Jaksch M, Hoyer S. Nerve growth factor and choline acetyltransferase activity levels in the rat brain following experimental impairment of cerebral glucose and energy metabolism. J Neurosci Res. 1992;31(3):479-86.

51. Bernhardi RV, Bernhardi LE, Eugenín J. Microglial cell dysregulation in brain aging and neurodegeneration. Front Aging Neurosci. 2015;7:1-21. 
Moreira PI, Carvalho C, Zhu X, Smith MA, Perry G. Mitochondrial dysfunction is a trigger of Alzheimer's disease pathophysiology. Biochim Biophys Acta. 2010;1802(1):2-10.

53. Castellani R, Hirai K, Aliev G, Drew KL, Nunomura A, Takeda A, et al. Role of mitochondrial dysfunction in Alzheimer's disease. J Neurosci Res. 2002;70(3):357-60.
54. Gibson GE, Sheu KFR, Blass JP. Abnormalities of mitochondrial enzymes in Alzheimer disease. J Neural Transm. 1998;105(8-9):855-70.

55. Levin ED. Nicotinic receptor subtypes and cognitive function. J Neurobiol. 2002;53(4):633-40.

56. Nordberg A, Winblad B. Reduced number of [3H]nicotine and $[3 \mathrm{H}]$ acetylcholine binding sites in the frontal cortex of Alzheimer brains. Neurosci Lett. 1986;72(1):115-20.
PICTORIAL ABSTRACT

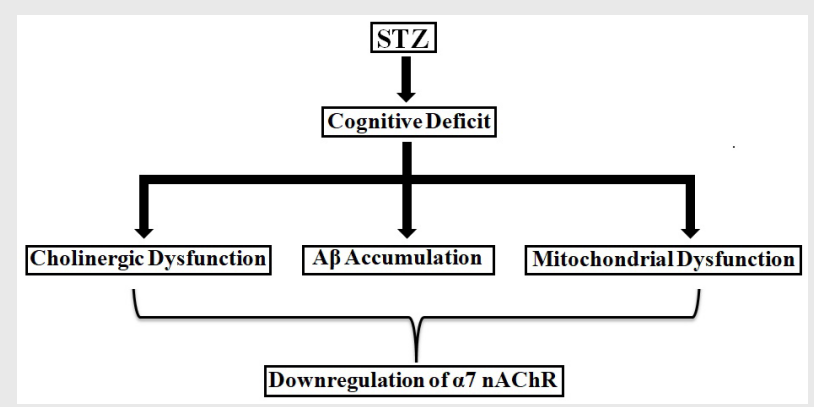

\section{SUMMARY}

- Intracerebroventricular infusion of streptozotocin into the lateral ventricles caused a downregulation in the $\alpha 7 \mathrm{nACh}$ receptor in the memory-sensitive rat hippocampus, pre frontal cortex (PFC) and amygdale.

- Intracerebroventricular streptozotocin infusion caused cholinergic dysfunction and accumulation of amyloid beta in these brain regions.

- Intracerebroventricular streptozotocin infusion impaired mitochondrial function, integrity and bioenergetics in these brain regions.

- The $\alpha 7$ nACh receptor could be a potential alternative target in the management of sporadic type of Alzheimer's disease.

\section{About Authors}

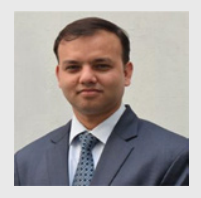

Niraj Kumar Singh, Assistant Professor, Division of Pharmacology, Institute of Pharmaceutical Research, GLA University, Mathura, 281406, India.

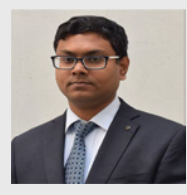

Debapriya Garabadu, Associate Professor, Division of Pharmacology, Institute of Pharmaceutical Research, GLA University, Mathura, 281406, India.

Cite this article: Singh NK, Garabadu D. Alpha7 Nicotinic Acetylcholine Receptor Down Regulation Impairs Mitochondrial Function in Streptozotocin-induced Sporadic Alzheimer's Disease Model in Rats. 2021;55(1):15363. 